\title{
Repurposing of Sildenafil as Hepatoprotective in Sinusoidal Obstruction Syndrome: Amendment of Endothelial Cell Damage and Inhibition of Platelet Aggregation
}

\author{
*Mona Mansour ${ }^{1}$, Amany M. Gad ${ }^{2,3}$, Heba S. Zaky ${ }^{1}$, Nayira A. Abdel Baky ${ }^{1}$ \\ ${ }^{1}$ Pharmacology and Toxicology Department, Faculty of Pharmacy (Girls), Al-Azhar University, Cairo, Egypt \\ 2 Department of Pharmacology, Egyptian Drug Authority (EDA), Formerly (NODCAR), Giza, Egypt, \\ ${ }^{3}$ Department of Pharmacology and Toxicology, Faculty of Pharmacy, Sinai University (East Kantara Branch), \\ New City, El Ismailia, Egypt \\ Correspondence: E-mail: : Mona.Mansour@azhar.edu.eg,mona_mansour90@yahoo.com,
} Telephone: +227296451

Article history: Received: 2021-04-2

Revised: 2021-05-14

Accepted: 2021-06-14

\begin{abstract}
Hepatic sinusoidal obstructive syndrome (SOS) is a rare drug-induced fibrous occlusive hepatic entity. Up to now, there is no perfect therapy to avoid SOS, therefore, new curative options are clearly needed. The current research pointed to explore the probable protective effect of sildenafil against monocrotaline (MCT)-induced SOS, and to further identify the underlying mechanisms. Sildenafil was given to rats for 7 days orally ( $5 \mathrm{mg} / \mathrm{kg} /$ day). In the fourth day animals fasted and given $90 \mathrm{mg} / \mathrm{kg} \mathrm{MCT}$. Twenty-four hours after last dose of sildenafil administration, liver tissue and blood were collected to evaluate SOS. Results revealed that sildenafil amended oxidative stress in liver tissue as indicated by the significant increase in hepatic GSH level. Furthermore, sildenafil treatment significantly decreased the expression of liver sinusoidal endothelial cell injury markers (hyaluronic acid (HA), Vascular Cell Adhesion Molecule-1 (VCAM-1), plasminogen activator inhibitor-1(PAI-1) and CD34, plus platelet aggregation markers (CD41 and P-selectin), as well as serum transaminases and hepatic caspase-3 activities. Additionally, macroscopic and histopathological examination of liver tissue augmented our biochemical findings. In conclusion, sildenafil markedly reversed the complicated and multifactorial pathophysiological state of SOS induced by MCT through its cytoprotective, thromboresistance effect on hepatic sinusoidal endothelial cells. Thereby, sildenafil proves to be a promising new therapeutic agent for SOS.
\end{abstract}

Key words: Sinusoidal obstruction syndrome; monocrotaline; hepatic sinusoidal endothelial cells; sildenafil

\section{INTRODUCTION}

Sinusoidal obstruction syndrome (SOS) is vascular hepatic problems characterized by damage in sinusoidal endothelium in zone 3 of liver acinus, that is associated with partial or full occlusion of small hepatic veins, therefore it has the previous term of hepatic veno-occlusive disease (VOD). It is one of the most horrific complications of cytoreductive therapies through hematopoietic stem cell transplantation (HSCT), that has more prevalence in pediatric compared to adults, with mortality rats reaching $80 \%$. Other SOS etiologies include adjuvant chemotherapy containing oxalipla tin , intake of medicinal herbs or tea -containing pyrrolizidine alkaloids, and using of tacrolimus in liver transplants ${ }^{1}$.

The pathophysiology of SOS/VOD is highly complicated and multifactorial. Severe depletion of glutathione (GSH) in hepatocytes is the inciting event in SOS incidence and increased risk. The toxic metabolites of chemotherapeutic agents that result from the hepatocellular cytochrome P450 metabolic process are mostly bio-transformed through conjugation with GSH to non-toxic metabolites. In zone 3 , the hepatocytes have elevated concentrations of cytochrome P450 and low levels of GSH, which cause these cells to be more liable to the damaging effect of these toxic agents and their metabolites ${ }^{2}$.

Hepatic sinusoidal endothelial cells (HSECs) are more sensitive than hepatocyte to toxic injury, and its activation considered an initial key that trigger multiple pathogenic pathways for $\mathrm{SOS}^{2}$. Activated HSECs release heparinase and increase expression of matrix metaloprotinase-9 (MMP-9) that cause breaking down of subcellular extracellular matrix proteins and loss of intercellular tight junctions ${ }^{3}$. These events also lead to intercellular gaps which increase vascular permeability and allow leakage of blood components into extravascular space of Disse causing incremental withdrawal of endothelial lining, aiding in narrowing and blockage in sinusoids. Activated HSECs further initiate inflammatory pathways through the expression and upregulation of inflammatory and adhesion molecules such as tumor necrosis factor- $\alpha(\mathrm{TNF} \alpha)$ and vascular cell adhesion

Cite this article: Mansour, M., Gad, A., Zaky, H., Abdel Baky, N. Repurposing of Sildenafil as Hepatoprotective in Sinusoidal Obstruction Syndrome: Amendment of Endothelial Cell Damage and Inhibition of Platelet Aggregation. Azhar International Journal of Pharmaceutical and Medical Sciences, 2021;1 (3):21 - 31. doi: 10.21608/aijpms.2021.206681 
molecule-1 (VCAM-1). The released cytokine and chemokine from activated cells cause further harm and damage to hepatocytes and HSECs. Damage to HSECs initiate also a hypercoagulable condition, with synthesis of tissue factor and plasminogen activator inhibitor-1(PAI-1) ${ }^{3}$. Both events contribute to platelet aggregation and stimulate activation of other clotting proteins within hepatic sinsiodes ${ }^{4}$, and result in more narrowing of venous lumen, reduced sinusoidal venous outflow, and post-sinusoidal portal hypertension. This cascade of events culminates in the creation of $\mathrm{SOS}^{3}$.

Animal models of SOS have greatly changed during the last 50 years in both their aim and methodology. Of these models, the monocrotaline (MCT)-induced model of SOS has been most extensivley utilized, especially in rats, which served as the basis for both novel diagnostic and therapy studies and has been modified over the last 20 years to optimize its use. MCT is part of the poisonous group of pyrrolizidine alkaloids (PAs), which are present in many species of Crotalaria plants ${ }^{5}$.

Management of SOS has only focused on symptomatic treatment, anticoagulant therapy, transjugular intrahepatic portosystemic shunt (TIPS), ending with liver transplantation depending on severity of the disease. The single FDA-approved therapy that is used only for severe cases is defibrotide, despite of its use as a prophylactic therapy, it remains under investigation ${ }^{6}$.

Sildenafil, a potent cyclic guanosine monophosphate (cGMP)-specific phosphodiesterase type 5 inhibitor, that commonly used to treat of erectile dysfunction ${ }^{7}$. Sildenafil does not only act as vasodilator, but also has additional actions on endothelial, vascular cells, and platelets ${ }^{8}$, by way of reduction of thromboxane $\mathrm{A}_{2}$, fibrinogen levels ${ }^{9}$, reduce expression of proinflammatory cytokine as $\mathrm{TNF} \alpha$ and interleukin-1 beta ${ }^{10}$, and inhibition of oxidative stress ${ }^{11}$. Additionally, data from several reports confirmed that, increased levels of cGMP prevent platelet aggregation, meanwhile inhibitors of type 5 phosphodiesterase prevent aggregation and adhesion of platelet in animal models ${ }^{12}$.

The current research was carried out to improve survival of SOS patients. We focused our thoughts on finding novel uses for an existing drug that is known as drug repositioning, via using sildenafil in treatment of MCT-induced SOS. Hopefully, we could find answers for research questions: if sildenafil has a promising hepatoprotective value against SOS caused by MCT and what are underlying mechanisms implicated in this protective process.

\section{METHODS}

\subsection{Animals:}

Adult male Sprague-Dawley rats, weighing 200 \pm 20 obtained from the Animal Homes of the
Egyptian Drug Authority (EDAs), formerly NDCAR, Giza, Egypt were used in this research. The rats housed in constant temperature $\left(23 \pm 1{ }^{\circ} \mathrm{C}\right)$, humidity $(60 \pm 10 \%)$, and a $12 / 12 \mathrm{~h} \mathrm{light/dark} \mathrm{cycle} \mathrm{at}$ controlled environmental conditions. They were adapted for 1 week prior to experimentation plus were allowed standard rat chow and water ad libitum. Animal Ethics Committee of the Faculty of Pharmacy, Al-Azhar University, Egypt, approved the experimental protocol used in this research (No. 203/2018).

\subsection{Drugs and Chemicals:}

Monocrotaline (MCT) and sildenafil (SLD) were obtained from Sigma-Aldrich Co. (St. Louis, MO, USA). All of the other chemicals used were of the highest commercially available quality.

\subsection{Experimental Design:}

A single dose of MCT $(90 \mathrm{mg} / \mathrm{kg})$ was given in the fourth day of the experiment to induce $\operatorname{SOS}^{13}$. The animals were fasted for $12 \mathrm{hr}$ before receiving MCT. Rats were randomly divided into 4 groups ( $n=6$ per group) and were treated according to the following scheme:

Group (1): Animals received saline oral via gavage for 7 days and considered as normal control group.

Group (2): Animals received saline oral via gavage for 7 days, and in the fourth day rats were fasted for $12 \mathrm{hr}$ then received MCT $(90 \mathrm{mg} / \mathrm{kg})$ orally by gavage once.

Group (3): Animals received $5 \mathrm{mg} / \mathrm{kg}$ sildenafil orally ${ }^{14}$ for 7 consecutive days and in the fourth day animals were fasted for $12 \mathrm{hr}$, then received MCT oral via gavage once.

Group (4): Animals received sildenafil oral via gavage for 7 days.

Animals were anaesthetized and blood samples were taken from the retro-orbital plexus twenty-four hours after the last dose of sildenafil was administered. For hematological analysis, some blood samples were collected in sterile specimen tubes containing EDTA, while for serum preparation, blood was collected in non-heparinized tubes and allowed to clot for 30 minutes at room temperature. After that, the serum was isolated by centrifugation at $3000 \mathrm{rpm}$ for 15 minutes and used to test liver functions. The rats were euthanized via cervical disclosure, and the liver tissues were removed, washed in ice cold saline, and blotted dry. For histopathological analysis, a sample of each liver tissue was fixed in $10 \%$ formalin. For the evaluation of various biochemical markers, other specimens were homogenized in phosphate buffered saline (PBS), $\mathrm{pH}=7.4$.

\subsection{Morphological Examination:}

Every group's animals were euthanized, then the abdominal organs were inspected through a midline incision. The livers were easily removed, rinsed with 
ice-cold phosphate-buffered saline ( $\mathrm{pH}$ 7.4) to remove any blood, blotted dry with a paper towel, and photographed. Each liver was examined macroscopically on the surfaces from all groups of the rats and the accumulation of peritoneal fluid and the color of the liver was recorded.

\subsection{Biochemical Analysis:}

\subsubsection{Hepatic oxidative stress markers}

Reduced glutathione (GSH) was measured by industrial kits (Biodiagnostic, Cairo, Egypt). The entire procedure was carried out in accordance with the manufacturer's instructions.

\subsubsection{Blood chemistry}

Red blood cells, white blood cells, hemoglobin, platelets, and neutrophils were counted using an automated blood cell counter (Celltac MEK-6458; NihonKohden, Tokyo, Japan).

\subsubsection{Enzyme-linked immunosorbent assay (ELISA)}

To obtain $10 \%$ homogenate, liver tissues were homogenized in ice-cold PBS $(\mathrm{pH}=7.4)$, then centrifuged for 15 minutes at 5,000 rpm and $4{ }^{\circ} \mathrm{C}$. The obtained supernatant was used to measure lactate dehydrogenase (LDH) (Catalog \# CSB-E11324r, Cusabio Biotech Co., Ltd.), vascular cell adhesion protein 1 (VCAM-1) (Catalog \# CSB-E07275r, Cusabio Biotech Co., Ltd.), plasminogen activator inhibitor 1 (PAI-1) (Catalog \# CSBE07948r,Cusabio Biotech Co., Ltd.), and caspase-3 (Casp-3) (Catalog \# E0280Ra, Bioassay Technology Laboratory, Shanghai, China), hyaluronic acid (HA) (Catalog \# MBS727090, MyBiosource, San Diego, CA, USA), sandwich ELISA kits were used according to the manufacturer's instructions.

2.5.4. Protein expressions of CD34, CD41 and pselectin using Western Blotting

Briefly, TRIzol reagent was used to extract CD34, CD41 and p-selectin proteins. The Bradford method was used to determine protein concentrations. SDS-PAGE gel electrophoresis was used to separate proteins, which were then transferred to nitrocellulose membranes. The primary antibodies used are for CD34 (Catalog \# PA5-89536, Thermo Fisher Scientific, USA), CD41 (Catalog \# PA5-79527, Thermo Fisher Scientific, USA), P-selectin (Catalog \# PA5-86855, Thermo Fisher Scientific, USA). A secondary horseradish peroxidase conjugated antibody (Thermo Fisher Scientific, USA; Catalog \# G-21234) was used to visualize the protein-antibody complex. X-ray films were used to capture the chemiluminescence produced. A luminescent image analyzer (FUJIFILM LAS-4000, Japan) was used to scan the films, and bands were quantified using Image J software. For CD34, CD41, and p-selectin, the results were expressed as arbitrary units after normalization for $\beta$-actin protein.

2.5.5. Assessment of liver biomarkers
Using commercially available kits (Spectrum diagnostics, Cairo, Egypt), the concentrations of alanine aminotransferase (ALT), aspartate aminotransferase (AST), albumin, and direct bilirubin in the blood were determined calorimetrically.

\subsection{Histopathological Examination}

Liver specimens were immediately fixed in $10 \%$ neutral buffered formalin, then dehydrated in ascending grades of ethanol, cleared in xylene and embedded in paraffin. Sections of three 4-micron thickness were cut and stained with hematoxylin and eosin before being studied under a light microscope 15. To avoid prejudices, all histopathologic steps and specimen evaluations were carried out by an experienced observer who was blinded to the identity of the samples examined.

\subsection{Statistical Analysis}

The mean \pm standard deviation (SD) are used to express the results. A one-way ANOVA followed by Tukey as post-hoc test were used for multiple comparisons. Statistical significance was described as a $\mathrm{p}$ value of less than 0.05 . Graph Pad Prism software version 5(San Diego, CA, USA) was being used for statistical analysis. Correlation coefficients were determined by linear regression analysis. The graphs were created with the Graph Pad Prism (version5).

\section{RESULTS}

\subsection{Sildenafil attenuated oxidative stress in MCT-} induced SOS in rat's liver.

Monocrotaline intoxication significantly heightened LDH levels by $333.2 \%$, when compared to control group. Meanwhile, sildenafil treatment returned LDH levels to normal values as compared with that of MCT group. Furthermore, MCT significantly reduced hepatic GSH content by $81.6 \%$ as compared to control group. while sildenafil treatment afforded significant protection against MCT intoxication and restored normal GSH content (Fig.1).

3.2. Sildenafil attenuated hepatic sinusoidal endothelial cell injury in MCT- induced SOS in rat.

Monocrotaline administration induced significant rise in hepatic HA by $478 \%$ and VCAM-1 levels by $210 \%$ associated with a significant elevation of PAI-1 content by $128 \%$ as compared to control group. PAI-1 act as endothelial injury marker that release following damage to HSEC and/or activated platelets nonetheless, sildenafil treatment completely abolished these effects (Table 1).

Moreover, protein expression of CD34 was assessed using western blot technique (Fig.2). which reflects sinusoidal capillarization in liver tissue and can be used to distinguish between healthy and damaged sinusoidal endothelium. MCT induced significant increase in CD34 expression by $441.5 \%$ 
in comparisons to control group. In contrast, a significant decline in sildenafil treated group was noticed.

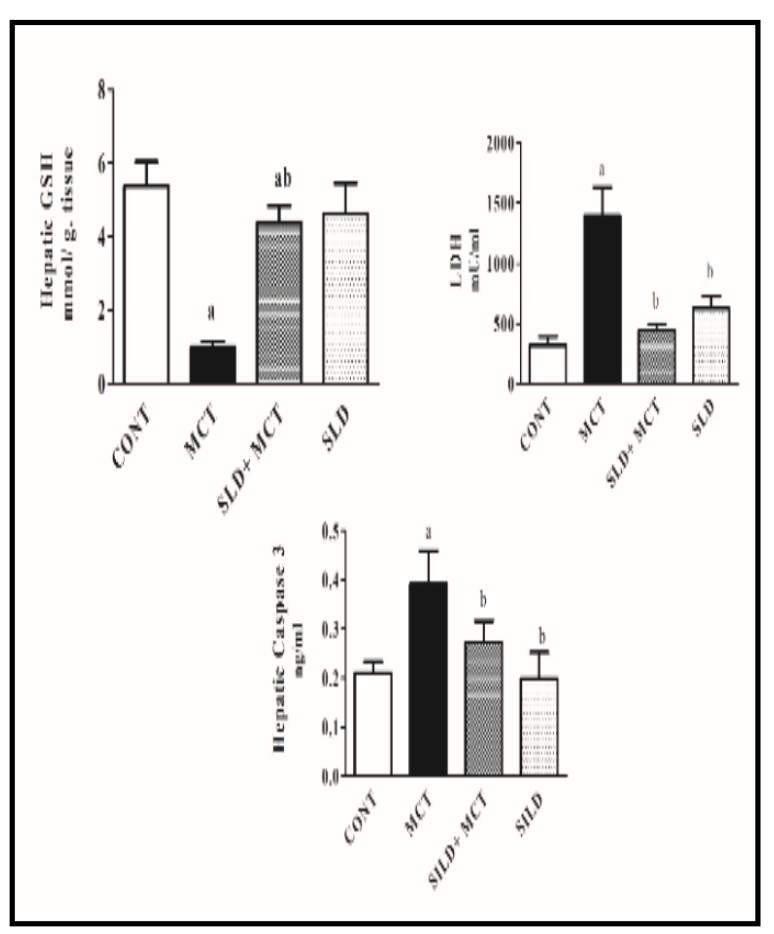

Figure 1. Sildenafil attenuated oxidative stress and apoptosis in MCT- induced SOS in rat's liver.

Each column reflects the mean of 6 animals \pm S.D. Oneway ANOVA was used for statistical analysis, followed by the Tukey- multiple comparison test as post-hoc test. ${ }^{a} \mathrm{p}<$ 0.05 vs control (CONT) group, ${ }^{b} \mathrm{p}<0.05 v s$ monocrotaline (MCT) group. Where, CONT; control, SLD; Sildenafil, MCT; monocrotaline, GSH; reduced glutathione, $\mathrm{LDH}$; lactate dehydrogenase, Casp-3; Caspase-3.

\subsection{Blood chemistry}

Administration of MCT showed a substantial decrease in hemoglobin, RBC counts and platelet counts by $48 \%, 31.5 \%, 37 \%$, respectively when compared to control group.

These changes were significantly attenuated in sildenafil treated group. However, WBC and neutrophils counts significantly increased in the MCT group by $215.2 \%$ and $283.6 \%$, respectively and these elevations were suppressed in the sildenafil treated group (Table).

\begin{tabular}{|l|l|l|l|}
\hline Groups & $\begin{array}{l}\text { HA } \\
\text { (ng/mg protein })\end{array}$ & $\begin{array}{l}\text { VCAM-1 } \\
\text { (ng/mg protein })\end{array}$ & $\begin{array}{l}\text { PAI-1 } \\
\text { (Pg/mg protein) }\end{array}$ \\
\hline CONT & $12.64 \pm 2.06$ & $32.74 \pm 2.45$ & $139.2 \pm 19.56$ \\
\hline MCT & $73.07 \pm 6.64^{\mathbf{a}}$ & $101.5 \pm 4.17^{\mathbf{a}}$ & $318 \pm 29.07^{\mathbf{a}}$ \\
\hline SLD+MCT & $31.07 \pm 4.60^{\mathbf{a} \mathbf{b}}$ & $52.85 \pm 2.92^{\mathbf{a} \mathbf{b}}$ & $193 \pm 6.14^{\mathbf{a} \mathbf{b}}$ \\
\hline SLD & $12.56 \pm 1.09^{\mathbf{b}}$ & $28.25 \pm 1.43^{\mathbf{b}}$ & $122.4 \pm 5.89^{\mathbf{b}}$ \\
\hline
\end{tabular}

Table 1. Effect of sildenafil on HA, VCAM-1 and PAI-1 in MCT induced SOS in rats.

Each values indicate the mean of 6 animals \pm S.D. One-way ANOVA was used for statistical analysis, followed by the Tukey- multiple comparison test as a post-hoc test. ${ }^{\mathrm{a}} \mathrm{p}<0.05$ vs the control (CONT) group, ${ }^{\mathrm{b}} \mathrm{p}<0.05$ vs the monocrotaline (MCT) group. Where, CONT; control, SLD; Sildenafil, MCT; monocrotaline, VCAM-1; Vascular cell adhesion molecule 1, PAI-1; Plasminogen activator inhibitors-1.

Table 2. The effect of sildenafil on hemoglobin level, RBC, platelet, WBC and neutrophils counts in MCT-induced SOS in rats.

\begin{tabular}{|c|c|c|c|c|c|}
\hline Groups & $\begin{array}{l}\text { Hemoglobin } \\
(\mathrm{g} / \mathrm{dL})\end{array}$ & $\begin{array}{l}\mathrm{RBC} \\
\left(10^{6} / \mu \mathrm{L}\right)\end{array}$ & $\begin{array}{l}\text { Platelet } \\
\left(10^{3} \mu \mathrm{L}\right)\end{array}$ & $\begin{array}{l}\text { WBC } \\
\left(10^{3} \mu \mathrm{L}\right)\end{array}$ & $\begin{array}{l}\text { Neutrophils } \\
\left(10^{3} \mu \mathrm{L}\right)\end{array}$ \\
\hline CONT & $15.98 \pm 0.76$ & 8. \pm 0.74 & $946.7 \pm 156.9$ & $5.51 \pm 1.081$ & $0.79 \pm 0.15$ \\
\hline MCT & $8.320 \pm 0.89^{a}$ & $5.59 \pm 0.40^{\mathrm{a}}$ & $596.0 \pm 39.29^{a}$ & $17.36 \pm 2.42^{\mathrm{a}}$ & $3.05 \pm 0.66^{\mathrm{a}}$ \\
\hline SLD+MCT & $16.02 \pm 0.95^{b}$ & $8.69 \pm 0.74^{b}$ & $932.2 \pm 141.0^{b}$ & $6.14 \pm^{\mathbf{b}} 1.16$ & $0.67 \pm 0.01^{\mathbf{b}}$ \\
\hline SLD & $14.30 \pm 1.51$ & $7.59 \pm 1.09$ & $904.0 \pm 118.7$ & $6.62 \pm 1.01$ & $0.72 \pm 0.10$ \\
\hline
\end{tabular}

Each value represents the mean of 6 animals \pm S.D. The one-way ANOVA was used in the statistical analysis, followed by the Tukey multiple comparison test as a post-hoc test $.{ }^{a} \mathrm{p}<0.05$ vs control (CONT) group, ${ }^{b} \mathrm{p}<0.05$ vs monocrotaline (MCT) group. Where, CONT; control, SLD; Sildenafil, MCT; monocrotaline, HB; hemoglobin, RBC; red blood cell, WBC; white blood cell. 
Table 3. Effect of sildenafil on ALT, AST, albumin, and direct bilirubin in MCT induced SOS in rats.

\begin{tabular}{|l|l|l|l|l|}
\hline \multicolumn{1}{|c|}{ Parameters } & $\begin{array}{l}\text { ALT } \\
(\mathrm{U} / \mathrm{mL})\end{array}$ & $\begin{array}{l}\text { AST } \\
(\mathrm{U} / \mathrm{mL})\end{array}$ & $\begin{array}{l}\text { Albumin } \\
(\mathrm{g} / \mathrm{dl})\end{array}$ & $\begin{array}{l}\text { Direct } \\
\text { bilirubin } \\
(\mathrm{mg} / \mathrm{dl})\end{array}$ \\
\hline CONT & $26.15 \pm 3.68$ & $71.2 \pm 11.9$ & $3.87 \pm 0.09$ & $1.49 \pm 0.30$ \\
\hline MCT & $144.2 \pm 30.84^{\mathbf{a}}$ & $112.0 \pm 13.10^{\mathbf{a}}$ & $2.08 \pm 0.46^{\mathbf{a}}$ & $2.19 \pm 0.51^{\mathbf{a}}$ \\
\hline SLD+MCT & $62.91 \pm 15.12^{\mathbf{a}} \mathbf{b}$ & $64.27 \pm 9.40^{\mathbf{b}}$ & $3.50 \pm 0.68^{\mathbf{b}}$ & $1.19 \pm 0.44^{\mathbf{b}}$ \\
\hline SLD & $18.53 \pm 3.52$ & $76.31 \pm 7.99$ & $3.84 \pm 0.18$ & $1.31 \pm 0.19$ \\
\hline
\end{tabular}

Each values indicate the mean of 6 animals \pm S.D. A one-way ANOVA study accompanied by Tukey Multiple Comparison tests as a post-hoc test were performed. ${ }^{\mathrm{a}} \mathrm{p}<0.05 v s$ control (CONT) group, ${ }^{\mathrm{b}} \mathrm{p}<0.05 v s$ monocrotaline (MCT)group. Where, CONT; control, SLD; Sildenafil, MCT; monocrotaline, ALT; alanine aminotransferase, AST; aspartate aminotransferase.

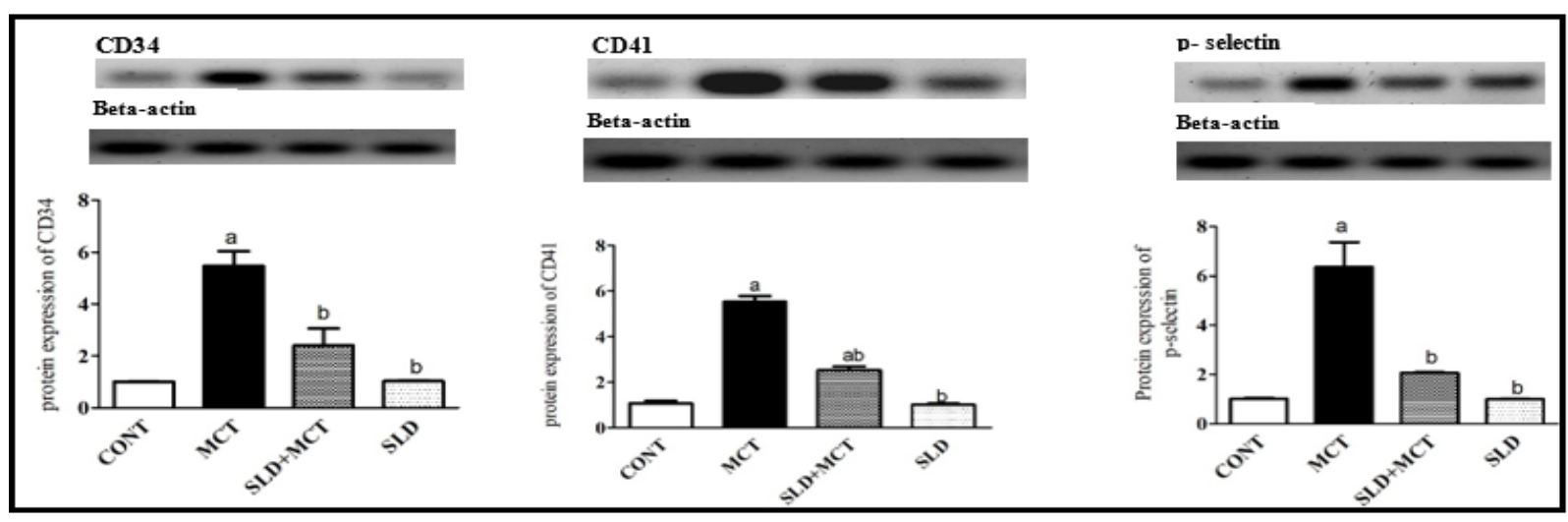

Figure2. Western blot analysis of CD34, CD41, and P-selectin expression in MCT-induced SOS in rats revealed a substantial increase that was attenuated by sildenafil therapy.

One-way ANOVA was being used for statistical analysis, followed by the Tukey- multiple comparison test as a post-hoc test. ${ }^{a} \mathrm{p}<0.05$ vs control (CONT) group, ${ }^{\mathrm{b}} \mathrm{p}<0.05$ vs monocrotaline (MCT) group. Where, CONT; control, SLD; Sildenafil, MCT; monocrotaline.

\subsection{Sildenafil attenuates platelet aggregation in MCT-induced SOS in the liver of rats.}

The effect of sildenafil on the platelet aggregation cascade was elucidated by studying its effect on CD41 protein expression and P-Selectin to detect activated platelets and damaged vascular endothelial cells proteins using western blot technique. MCT showed a sharp increase in liver CD41 and p-selectin expression by $417 \%$ and $523 \%$, respectively when compared to control. However, treatment with sildenafil prevented this increase to a large extent (Fig2).

\subsection{Histopathological Examination}

Microscopic examination to liver sections of control rats stained with $\mathrm{H} \& \mathrm{E}$ revealed that the central vein and precentral hepatocytes were normal histologically. In comparison, the MCT group demonstrated multiple focal necrosis and diffuse Kupffer cell activation as well as hemorrhages all over the parenchyma associated with extreme congestion in the hepatic sinusoids. Conversely, administration of sildenafil with MCT resulted in attenuation of the pathological changes induced by MCT in the liver where activated Kupffer and few necrobiotic changes of some hepatocytes were detected (Fig.3).

\subsection{Sildenafil attenuates apoptosis in MCT- induced SOS in rat's liver.}

Caspase-3 activity was significantly higher in the MCT group by $87.6 \%$ as compared to the control group. Surprisingly, this elevation was averted by sildenafil treatment (Fig.1).

\subsection{Liver Morphology}

At forth day after MCT administration, macroscopic liver findings in the MCT group revealed bloody peritoneal fluid, ascites, and the liver surface appeared congested and dark red relative to the control, indicating the production of SOS. These changes were less pronounced in the sildenafil-treated group (Fig. 4). but liver surface in sildenafil alone group appeared like control. 


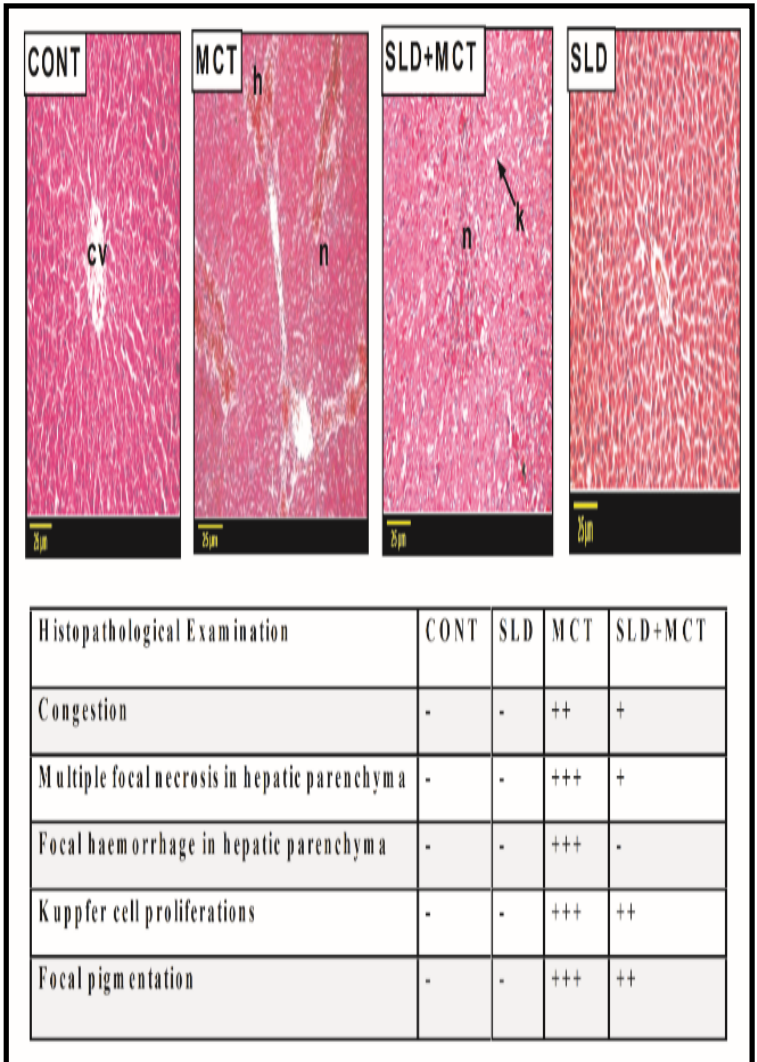

Figure 3. Histopathological examination of rat liver sections stained with $H \& E$ and grading of histopathological alterations in all treatment groups.

$\mathrm{CONT} \rightarrow$ Photomicrograph of control groups liver section viewing typical hepatic architecture, hepatocyte structure and central vein (CV). MCT $\rightarrow$ Photomicrograph of liver section of monocrotaline group showing multiple focal necrosis (n) with haemorrhages (h) in the parenchyma and showing severe congestion in sinusoids in focal manner. SLD + MCT $\rightarrow$ Photomicrograph of liver section of sildenafil co-treated group showing central vein dilatation while the parenchyma showed few focal necrosis (n) and diffuse Kupffer cells proliferation (k) were detected in between hepatocytes. SLD $\rightarrow$ Photomicrograph of sildenafil group only liver section display regular hepatic architecture and hepatocyte structure.

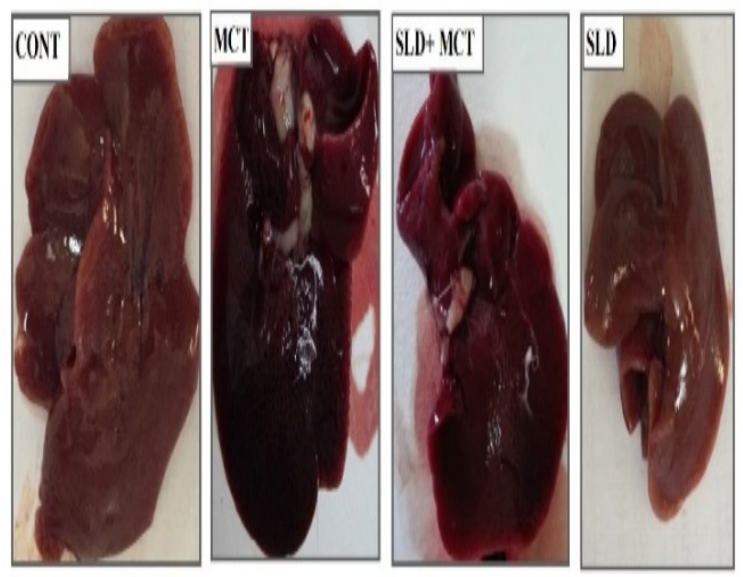

Figure 4. Macroscopic results in the normal livers of a control rat (CONT) plus a rat with MCT-induced SOS (MCT) and rat treated with sildenafil (SLD+ MCT).

MCT revealed a buildup of bloody ascites. Furthermore, the liver surface was dark red in colour, which was reduced in
(SLD+MCT) when sildenafil was combined with monocrotaline.

\subsection{Liver enzymes and liver function}

Monocrotaline administration induced marked hepatic damage proved by the significant elevations in serum ALT \& AST activities by $451 \%$ and $57.7 \%$ respectively, while, treatment with sildenafil decreased these elevations as compared to MCT group. Also, MCT disrupted the synthetic functions of the liver, as evidenced by the significant reduction serum albumin level by $46.29 \%$ and a significant increase in serum direct bilirubin by $45.70 \%$ as compared to CONT group. On the other hand, sildenafil treatment counteracted the reduction of albumin level and the elevation of direct bilirubin level as compared to MCT group (Table.3). These mean that after around $96 \mathrm{hr}$ or four days of MCT administration, hepatic damage has reached a maximum.

\subsection{Correlation Studies}

The estimation of ALT concentration as a specific indicator for liver damage induced by MCT in SOS rat liver was appeared to be directly correlated with the content of AST, albumin, GSH, LDH, HA, VCAM-1, PAI-1, HB, CD34, CD41, P-selectin and Caspase-3 ( $\mathrm{r}=0.8084, \mathrm{r}=-0.8169, \mathrm{r}=-0.8990, \mathrm{r}=$ $0.8427, r=0.9786, r=0.9819, r=0.9323, r=-0.9234$, $\mathrm{r}=0.9859, \mathrm{r}=0.9816, \mathrm{r}=0.9600$ and $\mathrm{r}=0.8501$ respectively, $\mathrm{p}<0.0001$ ) as shown in Fig. 5 (a-l).

GSH depletion was linked to an increased risk of SOS, so we calculated GSH levels and discovered that they were closely correlated with the content of ALT, AST, Albumin, LDH, HA, VCAM-1, PAI-1, HB, CD34, CD41, P-selectin and Caspase-3 $(\mathrm{r}=-0.8827$, $\mathrm{r}=-0.7893, \mathrm{r}=0.8686, \mathrm{r}=-0.8963, \mathrm{r}=-0.9154, \mathrm{r}=$ $0.9193, \mathrm{r}=-0.8967, \mathrm{r}=0.9119, \mathrm{r}=-0.9290, \mathrm{r}=$ $0.9286, \mathrm{r}=-0.9309$ and $\mathrm{r}=-0.8824$ respectively, $\mathrm{p}<$ $0.0001)$ as shown in Fig. 6 (a-1).

\section{DISCUSSION:}

Hepatic sinusoidal obstructive syndrome (SOS) is a rare fatal clinical disease that primarily evolves from injury in hepatic sinusoidal endothelial cells preceding coagulative necrosis of hepatic cells. Few therapeutic strategies are approved for SOS management ${ }^{1}$. Given the great importance of seeking medication for SOS, we highlighted the urgent need for new therapies to protect the liver and to increase the survival of SOS patients. The current research for the first-time sheds light on the promising hepatoprotective effect of sildenafil against SOS, and clarified its possible underlying mechanisms by analyzing its effect on oxidative stress, hepatic sinusoidal endothelial cells injury markers, blood chemistry, platelets aggregation markers, apoptosis as well as histopathological and morphological examination of liver tissue. 


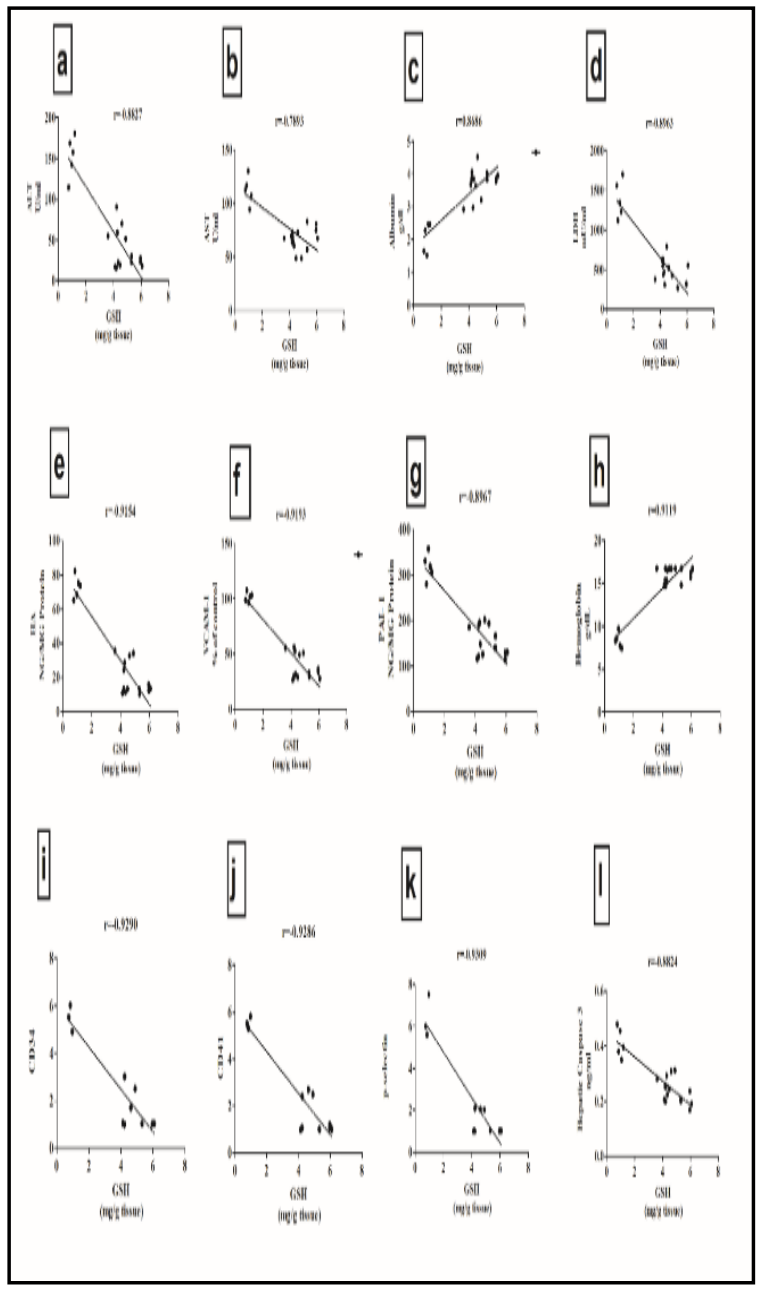

Figure 5. Correlation study and correlation coefficients between serum ALT level and AST (a), Albumin (b), GSH (c), LDH (d), HA (e), VCAM-1 (f), PAI-1 (g), HB (h), CD34 (i), CD41(j), P-selectin (k) and Caspase-3 (l) in liver tissues of MCT-induced SOS in rats. Linear regression analysis (0.0001) was used for statistical analysis.

To address our aim, MCT was used to induce SOS. MCT induces the same histological liver characteristics and similar symptoms like human SOS ${ }^{16}$, and it is considered as a reproducible model that facilitates studying the mechanism of the disease. MCT is a pyrrolizidine alkaloid that metabolized hepatically by cytochrome P450 into an alkylating agent. The reactive metabolite is dehydromonocrotaline, also called MCT pyrrole, that binds covalently to actin inside the endothelial cell ${ }^{17}$, is detoxified by conjugation with GSH. As GSH is the primary defense ligand in HSECs, the primary insult for MCT induced SOS is glutathione depletion ${ }^{18}$. In our study MCT significantly reduced hepatic GSH content and increased LDH level while treatment with sildenafil restored normal GSH content and reduced LDH levels to normal values ${ }^{14}$.

Another main event in MCT induced SOS that appeared in the first hours after MCT administration is the alteration of hepatic sinus

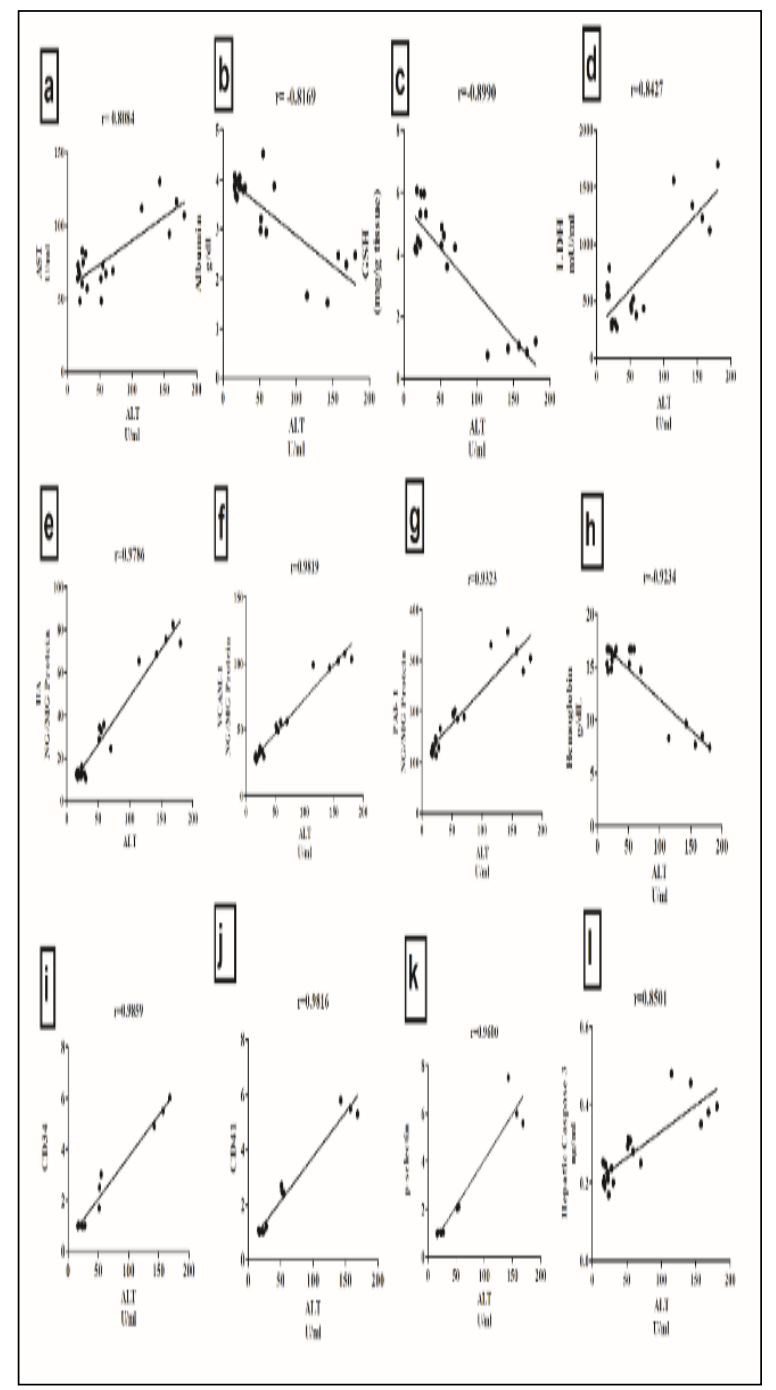

Figure 6. Analysis of Correlation and Correlation coefficients between serum GSH level and ALT (a), AST, (b) Albumin (c), LDH (d), HA (e), VCAM-1 (f), PAI-1 (g), HB (h), CD34 (i), CD41(j), P-selectin (k) and Caspase-3 (l) in liver tissues of MCT-induced SOS in rats. Linear regression analysis $<0.0001$ was being used for statistical analysis.

structure $^{13}$ and was documented in our result as a significant rise in hepatic HA level. Damage to HSECs impairs its ability to eliminate HA from the circulation, resulting in an increase in HA concentration ${ }^{4}$.Contrarily, administration of sildenafil counteracted MCT-induced elevation in HA level.

$\mathrm{CD} 34$, is an intercellular adhesion protein that is used to detect the presence of sinusoidal capillarization in liver tissue plus differentiating damaged and healthy sinusoidal epithelium ${ }^{19}$. In this study, MCT induced significant increase in CD34 
protein expression. Interestingly, treatment with sildenafil significantly decline hepatic CD34 protein expression in MCT treated rats. This finding is in the same line with the data of Hunt ${ }^{20}$ who reported that sildenafil manipulates sinusoidal endothelial cells fenestration in young and old liver.

Plasminogen activator inhibitors-1 serves as endothelial injury marker that release after injury in HSEC and/or activated platelets. It is used to diagnose and predict the severity of SOS ${ }^{21}$. In our study, MCT treatment induced a significant elevation in PAI-1 content, while sildenafil treatment completely abolished this effect. Our result is in accordance with other previous reports ${ }^{22}$. These data may signify the inhibitory effect of sildenafil on SOS development through protection of endothelial cells.

In various experimental models, MCT has been shown to increase oxidative stress and inflammation 23 .

Endothelial cell damage and vascular permeability are exacerbated by oxygen free radicalmediated inflammation ${ }^{24}$. Injured HSECs can attract white blood cells and adhere to inflammatory sites ${ }^{\mathbf{2 5}}$. VCAM-1 is one of the recruited adhesion molecules, and it plays an important role in the occurrence of SOS ${ }^{26}$. In our study, MCT treatment heightened hepatic VCAM-1 level, meanwhile, sildenafil treatment completely hampered this elevation. Our data is in line with Ibrahim ${ }^{27}$, who reported that, sildenafil reduced ICAM-1 and VCAM-1 levels in Alzheimer's disease rats. Sildenafil was documented to exhibit hepatoprotective effects by modulating antioxidant and inflammatory signaling pathways such as $\mathrm{P}_{38}-\mathrm{MAPK} / \mathrm{NF}-\mathrm{kB} / \mathrm{iNOS}$ and Keap-1/Nrf-2 28 .

Modification of HSECs morphology and destruction of sinusoidal lining disturb hepatic microcirculation by changes in HSEC morphology and the degradation of sinusoidal lining ${ }^{29}$. The concentration of hemoglobin in the blood was measured in order to quantify the degree of liver hemorrhage after MCT treatment. MCT treatment significantly decreased hemoglobin, platelet, and RBC counts. Meanwhile it markedly increased WBC and neutrophils counts. These changes were significantly attenuated in sildenafil treated group. These results are in line with those of other studies ${ }^{13}$. Aggregation of platelets in the extra-sinusoidal (Disse's) space and endothelial damage have been shown to play a significant role in SOS exacerbation ${ }^{13}$. The effect of sildenafil on the platelet aggregation cascade was studied by looking at how it affected the expression of the CD41 protein and P-selectin, which are used to detect activated platelets and damaged vascular endothelial cells. In the current study, CD41 and P-selectin expressions were elevated in the MCT group. Meanwhile, treatment with sildenafil reduced
CD41 and P-selectin expressions. Additionally, the count of platelets in the MCT group was significantly reduced, yet this reduction was significantly suppressed in sildenafil treated rats. These results were in keeping with the work done by ${ }^{13}$ and Mandosi ${ }^{30}$,who concluded that sildenafil reduces Pselectin through improving the endothelial dysfunction. Sildenafil has been demonstrated to improve vasodilatation and blood flow of tissue by relaxation of smooth muscles of the blood vessels, increasing new capillary formation, inhibiting platelet aggregation, and improving microcirculation.

Inhibition of apoptosis and activation of cellsurvival signal play an important function in maintaining the viability of endothelial cells ${ }^{31}$. MCT was shown to induce apoptosis in liver cells ${ }^{32}$. Our results agree with the aforementioned data, where MCT treatment significantly increased level of the apoptotic marker caspase- $3^{23}$. Treatment with sildenafil considerably decreased the activity of caspase-3 in MCT treated rats. By inhibiting apoptosis, sildenafil has been shown to alleviate postresuscitation myocardial and intestinal microcirculatory dysfunction ${ }^{33}$, as well as hepatocellular damage after liver ischemia reperfusion ${ }^{34}$ In CCl4-mediated hepatotoxicity, sildenafil also had a hepatoprotective role and induced liver regeneration ${ }^{35}$.

The histopathological examination of liver architecture verified our biochemical findings, as it revealed numerous focal necrosis with diffuse Kupffer cells activation and hemorrhages in all over the hepatic parenchyma associated with extreme congestion in the hepatic sinusoids of MCT treated group. Conversely, administration of sildenafil resulted in attenuation of these pathological findings induced by MCT in liver cells. This is line with the observations of ${ }^{36}$ who concluded that sildenafil prevents the progression of SOS in rats following oxaliplatin-based chemotherapy.

The protective effect of sildenafil was further mirrored in the macroscopic appearance of liver tissue in the sildenafil treated group, which showed much less hemorrhagic, congestion and ascetic fluid in the abdominal cavity as compared to the MCT treated group during first 96 hours of MCT administration. MCT-induced hepatic injury was further confirmed by the significant elevation in serum markers of hepatocellular injury as ALT and AST. These changes were further accompanied by a disruption in liver functions as evidenced from the marked reduction in serum albumin and concomitant increase in serum direct bilirubin. Treatment with sildenafil counteracted the aforementioned changes that was in line with data of previous reports ${ }^{37-38}$. 
Lastly, the current results documented 1) Normal liver architecture 2) MCT induced multiple cascade significant correlations between serum ALT activity involving depletion GSH, hepatocytes death, subsequently, and GSH level with AST, albumin, LDH, HA, activation of hepatic sinusoidal endothelial cell that upregulate VCAM-1, PAI-1, HB, CD34, CD41, P-selectin and adhesion of surface molecules and accompanied by alterations of caspase-3. In humans and preclinical research, serum coagulation pathways resulting in increase in HA, VCAM-1, ALT activity has been widely used as a significant PAI-1 level and expression of CD34, CD41, P-Selectin, biomarker for liver injury ${ }^{39}$, and GSH depletion has furthermore caspase-3 activation which contributes to apoptosis been suggested as a common mechanism causing and is involved in SOS pathogenesis. All these events leading to $\operatorname{SOS}^{40}$. Thus, we performed a correlation study for the development of hepatic sinusoidal obstruction, micro-vessel first time between these parameters. The present occlusion and marked hepatic damage proved by elevations in findings are similar with other previous studies that ALT \& AST activities and alteration in blood picture 3) sildenafil found clear associations between SOS pathology administration amended sinusoidal endothelial cell damage, score and ALT, AST, and direct bilirubin ${ }^{41}$. inhibited platelet aggregation and oxidative stress in liver tissue

In addition, Park ${ }^{42}$ reported that, injury of and restored normal hepatic architecture.

sinusoid and lobular inflammation grades were correlated to liver shear wave velocity (SWV). Likewise, a correlation was reported between ALT activity and other parameters that confirm hepatic damage in acute liver toxicity ${ }^{43-44}$.

\section{Conclusion:}

Conclusively, our experimental and correlational designs shed the light on a promising hepato protective action of sildenafil against hepatic SOS induced by MCT. Our results proved for the first time that, sildenafil markedly reversed this pathophysiological state through its hepatic sinusoidal endothelial cytoprotective, thromboresistance and antioxidant, effects. As the use of sildenafil increased hepatic GSH level and decreased platelet aggregation. A controlled clinical trial is needed to investigate the potential of these findings in patients with SOS.

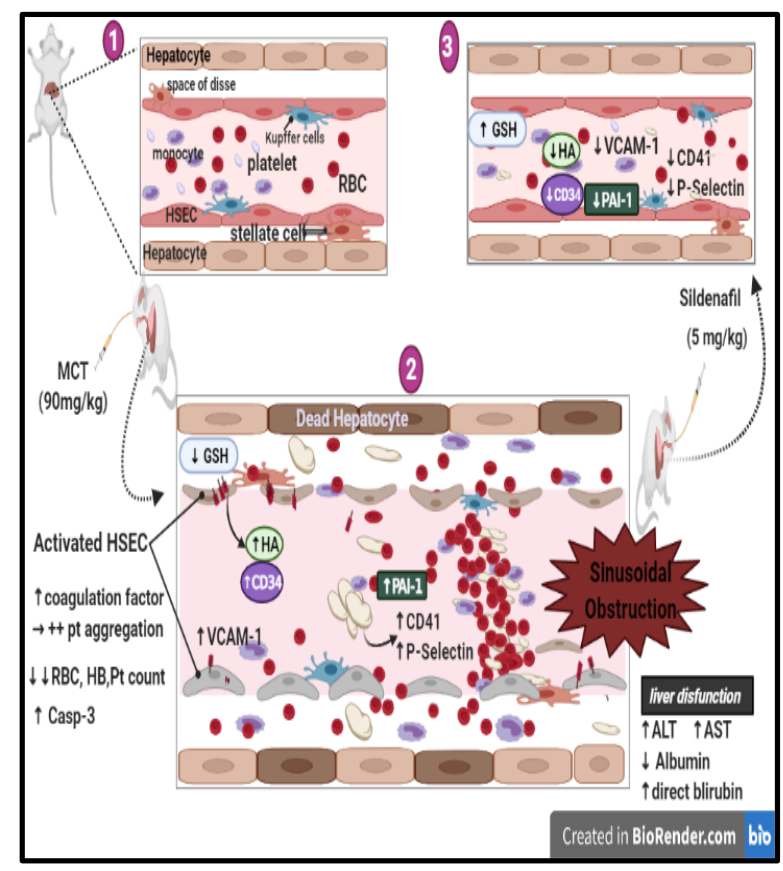

Figure 7. Graphical abstract recap the underlying mechanisms complicated in sildenafil hepatoprotective effect in SOS model.

\section{Acknowledgements:}

The authors would like to express their gratitude to the Egyptian Drug Authority (EDA), formerly the National Organization for Drug Control and Research (NODCAR), Giza, Egypt, for supplying the animals, chemicals, and kits used in this research. Prof. Dr. A. Bakear (Pathology Department, Faculty of Veterinary Medicine, Cairo University, Cairo, Egypt) deserves special thanks for her meticulous assistance with the histopathology.

\section{Ethics approval:}

The Ethics Committee at the Faculty of Pharmacy, Al-Azhar University (permit number: (No. 203/2018) approved all procedures used in this research. The inquiry followed the US National Institutes of Health's Guidelines for the Care and Use of Laboratory Animals (NIH Publication No. 85-23, revised 2011).

Conflicts of interest: There are no conflicts of interest reported by the authors.

Declarations: Not applicable.

Funding: This research was not funded by any specific, private, or non-profit funding agencies.

Authors' contributions: The research was conceived and designed by MM, AMG, HSZ, and NAA Experiments were carried out by MM, AMG. Data was analyzed by MM and AMG. The manuscript was written by MM, AMG, HSZ, and NAA. The final manuscript was read and accepted by all contributors. All data was collected at Al-Azhar University's Faculty of Pharmacy (Girls), Nasr City, Cairo, Egypt. List of abbreviations:

ALT; Alanine Aminotransferase, AST; Aspartate Aminotransferase, Casp-3;Caspase-3,cGMP; cyclic Guanosine Monophosphate, CONT; Control, GSH; Reduced Glutathione, HB; Hemoglobin, HSCT; Hematopoietic Stem Cell Transplantation, HSECs; Hepatic Sinusoidal Endothelial Cells, LDH; Lactate Dehydrogenase, MCT; Monocrotaline, MMP-9; Matrix Metaloprotinase-9, RBC; Red Blood Cell, SD; Standard Deviation, SLD ; Sildenafil, SOS; Sinusoidal Obstruction Syndrome, SWV; Liver Shear Wave 
Velocity, TIPS; Trans-Jugular Intrahepatic Portosystemic Shunt, TNFa; Tumor Necrosis Factor$\alpha$, VCAM-1; Vascular Cell Adhesion Molecule1,VOD; Veno-Occlusive Disease, WBC; White Blood Cell.

\section{REFERENCES:}

1. Fei Y, Peng Y, Sun H, Zou S, Yang J. Sinusoidal Obstruction Syndrome. Portal Hypertension: IntechOpen; 2021.

2. Fan CQ, Crawford JM. Sinusoidal Obstruction Syndrome (Hepatic Veno-Occlusive Disease). Journal of Clinical and Experimental Hepatology. 2014;4(4):332-346.

3. Cairo MS, Cooke KR, Lazarus HM, Chao N. Modified diagnostic criteria, grading classification and newly elucidated pathophysiology of hepatic SOS/VOD after haematopoietic cell transplantation. British journal of haematology. 2020

4. Copple BL, Banes A, Ganey PE, Roth RA. Endothelial cell injury and fibrin deposition in rat liver after monocrotaline exposure. Toxicological Sciences. 2002;65(2):309-318.

5. Kumar A, Palek R, Liska V. A critical analysis of experimental animal models of sinusoidal obstruction syndrome. Journal of clinical and experimental hepatology. 2019;9(3):345-353

6. de Lédinghen $\mathrm{V}$, Villate $\mathrm{A}$, Robin $\mathrm{M}$, Decraecker M, Valla D, Hillaire $S$, et al. Sinusoidal obstruction syndrome. Clinics and research in hepatology and gastroenterology. 2020;44(4):480-485.

7. Moreland RB, Goldstein I, Traish A. Sildenafil, a novel inhibitor of phosphodiesterase type 5 in human corpus cavernosum smooth muscle cells. Life Sciences. 1998;62(20):PL309-PL318.

8. Foresta C, De Toni L, Magagna S, Galan A, Garolla A. Phosphodiesterase-5 inhibitor tadalafil acts on endothelial progenitor cells by CXCR4 signalling. Current drug delivery. 2010;7(4):274282 .

9. El-Sayed M-IK, Amin HA-KA. Mechanism of endothelial cyto-protective and thromboresistance effects of sildenafil, vardenafil and tadalafil in male rabbit. Archives of medical science: AMS. 2015;11(1):190.

10. Tsai BM, Turrentine MW, Sheridan BC, Wang M, Fiore AC, Brown JW, et al. Differential effects of phosphodiesterase-5 inhibitors on hypoxic pulmonary vasoconstriction and pulmonary artery cytokine expression. The Annals of thoracic surgery. 2006;81(1):272-278.

11. Morano S, Mandosi E, Fallarino M, Gatti A, Tiberti C, Sensi M, et al. Antioxidant treatment associated with sildenafil reduces monocyte activation and markers of endothelial damage in patients with diabetic erectile dysfunction: a double-blind, placebo-controlled study. european urology. 2007;52(6):1768-1776.
12. Chiu PJ, Vemulapalli S, Chintala M, Kurowski $\mathrm{S}$, Tetzloff GG, Brown AD, et al. Inhibition of platelet adhesion and aggregation by E4021, a type $\mathrm{V}$ phosphodiesterase inhibitor, in guinea pigs. Naunyn-Schmiedeberg's archives of pharmacology. 1997;355(4):463-469.

13. Miyata T, Tajima H, Hirata M, Nakanuma Si, Makino I, Hayashi H, et al. Phosphodiesterase III inhibitor attenuates rat sinusoidal obstruction syndrome through inhibition of platelet aggregation in Disse's space. Journal of gastroenterology and hepatology. 2018;33(4):950957.

14. Molehin OR, Adeyanju AA, Adefegha SA, Aina OO, Afolabi BA, Olowoyeye AO, et al. Sildenafil, a phosphodiesterase- 5 inhibitor, offers protection against carbon tetrachloride-induced hepatotoxicity in rat. Journal of basic and clinical physiology and pharmacology. 2018;29(1):29-35.

15. Banchrof J, Steven A, Turner D. Theory and practice of histopathological techniques 4th ed. Churchil Livingstone, New York, London, San Francisco, Tokyo. 1996

16. DeLeve LD, Shulman HM, McDonald GB, editors. Toxic injury to hepatic sinusoids: sinusoidal obstruction syndrome (veno-occlusive disease). Seminars in liver disease; 2002: Copyright(C 2002 by Thieme Medical Publishers, Inc., 333 Seventh Avenue, New York, NY 10001, USA. Tel.:+ 1 (212) 584-4662.

17. Lamé MW, Jones AD, Wilson DW, Dunston SK, Segall H. Protein targets of monocrotaline pyrrole in pulmonary artery endothelial cells. Journal of Biological Chemistry. 2000;275(37):29091-29099.

18. Amin KA, Hashem KS, Al-muzafar HM, Taha EM. Oxidative hepatotoxicity effects of monocrotaline and its amelioration by lipoic acid, S-adenosyl methionine and vitamin E. Journal of Complementary and Integrative Medicine. 2014;11(1):35-41.

19. Couvelard A, Scoazec J-Y, Dauge M-C, Bringuier A-F, Potet F, Feldmann G. Structural and functional differentiation of sinusoidal endothelial cells during liver organogenesis in humans. 1996

20. Hunt NJ, Lockwood GP, Warren A, Mao H, McCourt PAG, Couteur DGL, et al. Manipulating fenestrations in young and old liver sinusoidal endothelial cells. American Journal of PhysiologyGastrointestinal and Liver Physiology. 2019;316(1):G144-G154.

21. Dignan FL, Wynn RF, Hadzic N, Karani J, Quaglia A, Pagliuca A, et al. BCSH/BSBMT guideline: diagnosis and management of venoocclusive disease (sinusoidal obstruction syndrome) following haematopoietic stem cell transplantation. British journal of haematology. 2013;163(4):444-457.

22. Hassan FM. The fibrinolytic alteration associated with daily administration of sildenafil. Asian Journal of Pharmaceutical and Clinical Research. 2018;11(8):107-109. 
23. Chang C-Y, Shih H-J, Huang I-T, Tsai P-S, Chen K-Y, Huang C-J. Magnesium Sulfate mitigates the progression of monocrotaline pulmonary hypertension in rats. International journal of molecular sciences. 2019;20(18):4622.

24. Clark CJ, Boswell F, Greer IA, Lyall F. Treatment of endothelial cells with serum from women with preeclampsia: effect on neutrophil adhesion. Journal of the Society for Gynecologic Investigation. 1997;4(1):27-33.

25. Pober JS, Sessa WC. Evolving functions of endothelial cells in inflammation. Nature Reviews Immunology. 2007;7(10):803-815.

26. Carreras E, Díaz-Beyá M, Rosiñol L, Martínez $\mathrm{C}$, Fernández-Avilés $\mathrm{F}$, Rovira $\mathrm{M}$. The incidence of veno-occlusive disease following allogeneic hematopoietic stem cell transplantation has diminished and the outcome improved over the last decade. Biology of blood and marrow transplantation. 2011;17(11):1713-1720.

27. Ibrahim M, Haleem M, AbdelWahab S, Abdel-Aziz A. Sildenafil ameliorates Alzheimer disease via the modulation of vascular endothelial growth factor and vascular cell adhesion molecule-1 in rats. Human \& Experimental Toxicology. 2020:0960327120960775.

28. Ali FE, Azouz AA, Bakr AG, Abo-Youssef AM, Hemeida RA. Hepatoprotective effects of diosmin and/or sildenafil against cholestatic liver cirrhosis: The role of Keap-1/Nrf-2 and P38MAPK/NF- $\kappa$ B/iNOS signaling pathway. Food and Chemical Toxicology. 2018;120:294-304.

29. DeLeve LD, editor Hepatic microvasculature in liver injury. Seminars in liver disease; 2007: C Thieme Medical Publishers.

30. Mandosi E, Giannetta E, Filardi T, Lococo M, Bertolini C, Fallarino M, et al. Endothelial dysfunction markers as a therapeutic target for Sildenafil treatment and effects on metabolic control in type 2 diabetes. Expert opinion on therapeutic targets. 2015;19(12):1617-1622.

31. Surico D, Farruggio S, Marotta P, Raina G, Mary D, Surico N, et al. Human chorionic gonadotropin protects vascular endothelial cells from oxidative stress by apoptosis inhibition, cell survival signalling activation and mitochondrial function protection. Cellular Physiology and Biochemistry. 2015;36(6):2108-2120.

32. Copple BL, Rondelli CM, Maddox JF, Hoglen NC, Ganey PE, Roth RA. Modes of cell death in rat liver after monocrotaline exposure. Toxicological Sciences. 2004;77(1):172-182.

33. Zhang Q, Wang G, Yuan W, Wu J, Wang M, Li C. The effects of phosphodiesterase-5 inhibitor sildenafil against post-resuscitation myocardial and intestinal microcirculatory dysfunction by attenuating apoptosis and regulating microRNAs expression: essential role of nitric oxide syntheses signaling. Journal of translational medicine. 2015;13(1):1-15.

34. Savvanis S, Nastos C, Tasoulis M-K, Papoutsidakis N, Demonakou M, Karmaniolou I, et al. Sildenafil attenuates hepatocellular injury after liver ischemia reperfusion in rats: a preliminary study. Oxidative medicine and cellular longevity. 2014;2014

35. Yardimci S, Bostanci E, Ozer I, Dalgic T, Surmelioglu A, Aydog G, et al., editors. Sildenafil accelerates liver regeneration after partial hepatectomy in rats. Transplantation proceedings; 2012: Elsevier.

36. Bostanci MT, Yilmaz İb, Saydam M, Uzunoğlu H, Aydog G, Koşmaz K, et al. The protective effect of sildenafil on liver sinusoidal obstructive syndrome after oxaliplatin-based chemotherapy: An experimental animal study. Journal of Surgery and Medicine. 2020;4(7):587591.

37. Abd El Motteleb DM, Ibrahim IA-H, Elshazly SM. Sildenafil protects against bile duct ligation induced hepatic fibrosis in rats: potential role for silent information regulator 1 (SIRT1). Toxicology and Applied Pharmacology. 2017;335:64-71.

38. Baran AH, Berk A, Kaymaz MB, Aktay G. Antioxidant Effect of Sildenafil on CadmiumInduced Liver, Lung and Kidney Injury. FABAD Journal of Pharmaceutical Sciences. 2020;45(1):37-44.

39. Yang X, Schnackenberg LK, Shi Q, Salminen WF. Hepatic toxicity biomarkers. Biomarkers in Toxicology: Elsevier; 2014. p. 241-259.

40. Wang X, Kanel GC, DeLeve LD. Support of sinusoidal endothelial cell glutathione prevents hepatic veno-occlusive disease in the rat. Hepatology. 2000;31(2):428-434.

41. Shin J, Yoon H, Cha YJ, Han K, Lee M-J, Kim $\mathrm{M}-\mathrm{J}$, et al. Liver stiffness and perfusion changes for hepatic sinusoidal obstruction syndrome in rabbit model. World Journal of Gastroenterology. 2020;26(7):706.

42. Park SH, Lee SS, Sung J-Y, Na K, Kim HJ, Kim SY, et al. Noninvasive assessment of hepatic sinusoidal obstructive syndrome using acoustic radiation force impulse elastography imaging: A proof-of-concept study in rat models. European radiology. 2018;28(5):2096-2106.

43. Abdelaziz AI, Mantawy EM, Gad AM, Fawzy $\mathrm{HM}$, Azab SS. Activation of pCREB/Nrf-2 signaling mediates re-positioning of liraglutide as hepato-protective for methotrexate-induced liver injury (MILI). Food and Chemical Toxicology. 2019;132:110719.

44. Ahmed LA, Abd El-Rhman RH, Gad AM, Hassaneen SK, El-Yamany MF. Dibenzazepine combats acute liver injury in rats via amendments of Notch signaling and activation of autophagy. Naunyn-Schmiedeberg's Archives Pharmacology. 2020:1-12. 